\title{
ASSOSYMMETRIC RINGS
}

\author{
ERWIN KLEINFELD ${ }^{1}$
}

1. Introduction. A nonassociative ring, sometimes called ring for short, does not presuppose the associative law of multiplication. However in virtually all the studies of such rings, while it is true that the full associative law of multiplication is not assumed, invariably some form of it is, so that strictly speaking the associative law of multiplication has not been done away with; it has merely been weakened. Alternative, commutative, anti-commutative, and thereby Jordan and Lie rings are flexible, that is to say satisfy the identity $(x, y, x)$ $=0$, where $(x, y, z)=x y \cdot z-x \cdot y z$. While right-alternative rings in general are not flexible, those of characteristic not 2 as well as alternative, Jordan and Lie rings are power-associative, that is satisfy the condition that every subring generated by a single element is associative. In the present study a class of rings is considered which come very close to being associative. Interestingly enough they are neither flexible nor power-associative. For lack of a better name these rings will be called assosymmetric. By definition an assosymmetric ring is one in which the associative law of multiplication has been weakened to the condition that $(P(x), P(y), P(z))=(x, y, z)$, for every permutation $P$ of $x, y, z$. Examples of assosymmetric rings which are not associative appear at the end of the paper. The main result is the following:

THEOREM. If $R$ is an assosymmetric ring without ideals $I \neq 0$, such that $I^{2}=0$, then $R$ is associative, provided the characteristic of $R$ is different from 2 and 3.

The restriction on the characteristic turns out to be necessary, for there exist even simple, assosymmetric rings of characteristic 2 and 3 which are not associative.

2. Identities. In any ring the identity

$$
(w x, y, z)-(w, x y, z)+(w, x, y z)=w(x, y, z)+(w, x, y) z,
$$

holds. Define $f(w, x, y, z)$, as in the case of an alternative ring [1], by means of

Received by the editors August 20, 1956.

1 This work was supported in part by the Office of Naval Research under contract ONR-609(19) with Yale University. 


$$
f(w, x, y, z)=(w x, y, z)-x(w, y, z)-(x, y, z) w .
$$

One discovers shortly that $f(w, x, y, z)=0$ in any assosymmetric ring. Note that the right hand side of (1) may be written as $(z w, x, y)$ $-f(z, w, x, y)$, so that

(3) $f(z, w, x, y)=(z w, x, y)-(w x, y, z)+(x y, z, w)-(y z, w, x)$.

Now it follows readily from (3) that

$$
f(w, x, y, z)=-f(z, w, x, y) \text {. }
$$

Up to this point all identities, as well as the proofs, apply to alternative rings as well. The next identity does not, however. It can be seen from (2) that

$$
f(w, x, y, z)=f(w, x, z, y) .
$$

But then $f(w, x, y, z)=-f(z, w, x, y)=-f(z, w, y, x)=f(x, z, w, y)$ $=f(x, z, y, w)=-f(w, x, z, y)=-f(w, x, y, z)$, using (5) and (4) alternately. Thus $2 f(w, x, y, z)=0$. We assume henceforth that we are in an assosymmetric ring of characteristic different from 2 and 3. Thence

$$
f(w, x, y, z)=0 .
$$

If each of the four terms of the right hand side of (3) is broken up by means of (2), then because of (6) the identity

$$
(w,(x, y, z))-(x,(y, z, w))+(y,(z, w, x))-(z,(w, x, y))=0,
$$

is obtained, where $(a, b)=a b-b a$. On the other hand if one breaks up $((x, w), y, z)+((z, y), w, x)$ by means of (2) one also obtains the left hand side of $(7)$. Consequently

$$
((x, w), y, z)+((z, y), w, x)=0 .
$$

Interchanging $w$ and $x$ in (8) and adding the result to (8) one obtains $2((z, y), w, x)=0$, so that

$$
((z, y), w, x)=0 .
$$

In an arbitrary ring the identity

$$
(t, y, z)+(y, z, t)+(z, t, y)=(t y, z)+(y z, t)+(z t, y)
$$

can readily be verified. By forming the associators of each side of (10) with $w$ and $x$, and using (9) it follows that $3((t, y, z), w, x)=0$. Thus

$$
((t, y, z), 7 w, \ldots x)=0
$$


Using (11), one forms the associators of both sides of (1) with $s$ and $t$, thus obtaining

$$
(w(x, y, z), s, t)+((w, x, y) z, s, t)=0 .
$$

On the other hand by interchanging $y$ and $z$ in (12) and subtracting the result from (12) one obtains

$$
((w, r x ; y) z, s, t)=((w, x, z) y, s, t) .
$$

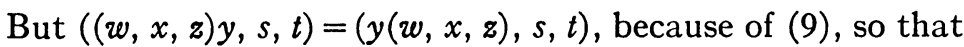

$$
((w, x, y) z, s, t)=(y(w, x, z), s, t),
$$

as a result of (13). Also by permuting $w$ and $y$ in (12) one obtains $(y(w, x, z), s, t)+((w, x, y) z, s, t)=0$. Comparison of this last identity with (14) yields $2((w, x, y) z, s, t)=0$. Therefore

$$
((w, x, y) z, s, t)=0 \text {. }
$$

Expanding (15) in the manner of (1), with the help of (11), now yields

$$
(w, x, y)(z, s, t)=0 \text {. }
$$

Although it is possible to derive further interesting identities we stop at this point, since the main result can now be established.

3. Main results. We are ready to prove the following:

THEOREM. If $R$ is a ring satisfying identities (11) and (16), has characteristic different from 2 and 3 , and has no ideals $I \neq 0$ such that $I^{2}=0$, then $R$ is associative.

Proof. In any ring $R$ the set $S$ of all finite sums of elements of the form $(R, R, R)$ and $(R, R, R) R$ is an ideal of $R . S$ is in fact the smallest ideal modulo which $R$ is associative. We shall be done if we can show that $S^{2}=0$. This entails proving that the product of every two elements of $S$ is zero. Let us first look at (11). Then it is clear, in view of (16), that we are done if we establish $(r, s, t) w(x, y, z)=0$, for all elements $r, s, t, w, x, y, z$ of $R$. But $w(x, y, z)=(w x, y, z)-(w, x y, z)$ $+(w, x, y z)-(w, x, y) z$ because of (1). Multiplying this equation through by $(r, s, t)$ on the left, with the aid of (11) and (16), it becomes obvious that $(r, s, t) w(x, y, z)=0$. But then $S^{2}=0$, so that $S=0$, and hence $R$ is associative. This completes the proof.

The theorem stated in the introduction, that an assosymmetric ring of characteristic different from 2 and 3 , and without ideals $I \neq 0$, such that $I^{2}=0$ is associative, is a direct consequence of the theorem just established. For it was shown that (11) and (16) hold 
in any assosymmetric ring of the desired characteristic. We conclude with several examples of assosymmetric rings which are not associative.

Example 1. Consider the algebra having basis elements $x, y, z$ over an arbitrary field. We define $x^{2}=y, y x=z$, and all other products of basis elements equal to zero. Thus all associators involving basis elements are zero except $(x, x, x)=z$. It is thus clear that this algebra is assosymmetric, but neither flexible nor power-associative.

EXAMPLE 2. Any alternative ring of characteristic 2 is obviously assosymmetric. Consequently the Cayley-Dickson algebras of characteristic 2 serve as examples of simple, assosymmetric but not associative algebras. We note also that an assosymmetric algebra of characteristic two need not be alternative. This is illustrated by the algebra of Example 1 over a field of characteristic 2.

Example 3. Consider the algebra with basie elements $1, x, y$ over a field of characteristic 3 , where 1 is the unit element and $x^{2}=y$, $y^{2}=2 x, x y=1, y x=0$. It may be verified that this is a simple, assosymmetric algebra. Since $(x, x, x)=-1$, the algebra is neither flexible nor power-associative.

\section{BIBLIOGRAPHY}

1. R. H. Bruck and Erwin Kleinfeld, The structure of alternative division rings, Proc. Amer. Math. Soc. vol. 2 (1951) pp. 878-890.

YALE UNIVERSITY 